\title{
MiR-99a-5p regulates proliferation, migration and invasion abilities of human oral carcinoma cells by targeting NOX4
}

 \\ ${ }^{1}$ Department of Stomatology, Dongfeng General Hospital affiliated with Hubei University of Medicine, Shiyan, 442000, Hubei, China; ${ }^{2}$ Department \\ of Stomatology, Dongying People's Hospital, Dongying, 257091, Shandong, China \\ *Correspondence: caic1888@163.com
}

Received December 3, 2016 / Accepted January 28, 2017

\begin{abstract}
Previous research has showed that miR-99a-5p was a tumor suppressor. The aim of our study was to explore the effect of miR-99a-5p on the vitality and proliferation, migration together with the invasion of oral tumor cells via inhibiting the expression of NOX4. QRT-PCR and Western blot were applied to examine the expression level of miR-99a-5p and NOX4 in human oral tumorous and adjacent tissues. Dual luciferase reporter gene assay was applied to confirm that miR-99a-5p negatively regulated directly on NOX4 in TSCC1 cells. Cell transfection and lentiviral vectors were used to up-regulate expression of miR-99a-5p and NOX4, respectively. Cell proliferation, cell cycle, apoptosis and invasion along with the migration in different groups were assessed using MTT assay, colony formation assay, the flow cytometry, transwell assay and the wound healing assay, respectively. MiR-99a-5p was under-expressed in human oral tumor, while NOX4 was over-expressed. There was a negative relationship between miR-99a-5p and NOX4. Up-regulating miR-99a-5p or down-regulating NOX4 suppressed the vitality, proliferation, migration together with invasion of TSCC1 cells. MiR-99a-5p affected the vitality and proliferation, migration together with the invasion of oral tumor cells through targeting NOX4.
\end{abstract}

Key words: oral carcinoma, miR-99a-5p, NOX4

Oral squamous cancer carcinoma (OSCC), one of the most prevalent malignancies, affects 300,000 individuals per year worldwide [1]. Among all types of head and neck cancers, OSCC is the most frequent one with a percentage at more than $90 \%$ [2]. Despite the fact that surgery, chemotherapy and radiotherapy have been developed for the treatment, OSCC continues to show a poor prognosis and remains lethal for nearly $50 \%$ of cases diagnosed annually [3]. Therefore, the therapeutic strategies for OSCC remain challenging and urgent.

MicroRNAs (miRNAs) are a group of non-coding smalllength RNAs that negatively regulate gene expressions, i.e. degrade their target mRNAs or hinder the translation of mRNAs, by binding to the 3 '-untranslated region (3'-UTR) of a mRNA [4]. MiRNAs have been reported to regulate the progression of various diseases. For instance, miR-125a has an important role in therapeutic intervention of OSCC and other cancers by targeting ESRRA [5] whereas miR-375 can bind directly to the 3'-UTR of KLF5, encoding a significant transcription factor and suppress the growth of OSCC tumors [6]. Moreover, miR-99a down-regulation was also confirmed in oral cancer cell lines and ectopic miR-99a expression was demonstrated to exert inhibition on oral cancer cell migration and invasion [7]. Interestingly, the corresponding role and function of other members of miR-99a family, e.g. miR-99a-5p have not been identified yet.

Previous studies have suggested the central role of NADPH oxidase in several diseases, which was associated with the regulation of different miRNAs. NOX4 could be silenced by miR-25 in diabetic nephropathy [8]. NOX4 was reported to be reduced by miR-23a in reducing the neuropathic pain, where NOX4 acted as an inflammation-promoting factor for neural cells after traumatic injury [9]. It was also presented that $N O X 4$ would play a growth-inhibitory role in liver cancer cells [10], implying its potential function in the intervention of various human cancer. From TargetScan databases, we found that NOX4 may be the target gene of miR-99a-5p, so we hypothesized that there was association between miR-99a-5p, NOX4 and OSCC.

Our study investigated the expression pattern of miR-99a-5p and NOX4 in OSCC cells and their effects on OSCC cell lines, aiming to reveal the underlying mechanism through which miR-99a-5p affects OSCC pathogenesis and provide useful information for the development of OSCC therapeutic strategies. 


\section{Materials and methods}

Tissue sample. The oral tumor tissues and pericarcinous tissues $(n=20)$ obtained from department of stomatology in Dongfeng General Hospital affiliated with Hubei University of Medicine during 2012 to 2015 were used in the present study. Pathological examination was used to examine the oral tumor tissues and cells which have not been treated with chemoradiotherapy or targeted therapy. Tissue samples were collected in operation and saved in $-80^{\circ} \mathrm{C}$. Informed consent had been obtained from all patients, and the research protocols have been approved by the Ethics Committee of Xiangya Hospital.

Cell transfection. (1) Roswell Park Memorial Institute 1640 (RPMI-1640) medium supplemented with $100 \mathrm{U} / \mathrm{ml}$ penicillin, $100 \mathrm{U} / \mathrm{ml}$ streptomycin and $10 \%$ fetal bovine serum (FBS) was applied to routinely incubate TSCC1 cell lines (BNBIO, Beijing, China), which were then placed in an incubator with $5 \% \mathrm{CO}_{2}$ at $37^{\circ} \mathrm{C}$.

(2) According to the manufacturer's instructions, Lipofectamine $^{\mathrm{TM}} 2000$ reagent was used to transfect miR-99a-5p mimics, miR mimics negative control (NC) and NOX4 siRNAs (with the final concentration $100 \mathrm{nM}$ ) into TSCC1 cells $\left(1.5 \times 10^{5}\right.$ cells each group). The mimics, negative controls and the siRNAs were all synthesized by Shanghai GenePharma Company (the sequences were listed in Table 1).

(3) NOX4 cDNA (GenePharma, Shanghai, China) was inserted into the lentiviral vector (pLenti-GIII-UbC). The recombinant vector, plenti-GIII-Ubc-NOX4 $(1 \mu \mathrm{g} / \mathrm{ml})$, was transfected into cells $\left(1.5 \times 10^{5}\right.$ cells each group) later to over-express NOX4.

Immunohistochemical analysis. Streptavidin-peroxidase (SP) immunohistochemical staining method was adopted to determine the expressions of SOX4 in tissue samples. In accordance with the kit (Maxin, China), rabbit anti-human SOX4 antibodies (dilution 1:1000, BD, USA) were used as primary antibodies. PBS buffer instead of the primary antibody was utilized as a negative control. Horseradish peroxidaseconjugated (HRP-conjugated) goat anti-rabbit IgG antibody (dilution 1:800, Zhongshan Biology Company, Beijing) was used as the secondary antibody. Then samples were analyzed by 3, 3'-diaminobenzidine (DAB) substrate kit (Maxin, China) according to the manufacturer's instructions.

Quantitative Real-Time PCR (qRT-PCR). According to the manufacturer's instructions, TRIzol reagent (TaKaRa company, Dalian, China) and spectrophotometer were used to extract the entire RNA and measure concentration of samples successively. The RNA was inverse-transcribed into cDNA and stored at $-20^{\circ} \mathrm{C}$ for the following use. Subsequently, cDNA was used as the template to detect the expression level of the miR-99a-5p and NOX4 mRNA after amplification via PCR (the primer sequences were shown in Table 2). U6 small RNA was applied as an internal standard for the normalization of miR-99a-5p, while GAPDH was for NOX4 mRNA. The $2^{-\Delta \Delta C_{t}}$ method was used for data analysis.

Dual luciferase reporter gene assay. To construct the pGLO-NOX4-3'UTR-wt and pGLO-NOX4-3'UTR-mut recombinant vectors, wild-type and mutant NOX4 3'UTR (Sangon Biotech, Shanghai, China) were inserted into the locus between SacI and Sal I of Dual-Luciferase miRNA Target Expression Vector (Promega, USA), which was then sequenced to confirm that there is no mutation in the inserted sequence (relative primer sequences were shown in Table 3). Subsequently, the cells were co-transfected with the recombi-

Table 1. The sequence information for miR-99a-5p mimics, NC and NOX4 siRNA

\begin{tabular}{ll}
\hline Name & Sequence \\
\hline MiR-99a-5p mimics & 5'-CGAACCCGTAGATCCGATCTTGTGGTTTTGGCCACTGACCACAAGATGATCTACGGGTTCA-3' \\
NC & 5'-CGAAATGTACTGCGCGTGGAGACGTTTTGGCCACTGACTGACGTCTCCACGCAGTACATTTCA-3' \\
NOX4 siRNA & 5'-CCUGGCCAGUAUAUUAU-3' \\
\hline
\end{tabular}

Table 2. The sequence of primers for RT-PCR

\begin{tabular}{lll}
\hline cDNA & Forward primer & Reverse primer \\
\hline MiR-99a-5p & 5'-AACCCGUAGAUCCGAUCUUGUG-3' & \\
NOX4 & 5'-GGAGTTGACGTCGGAAT-3' & 5'-AGTAACTTCGACTTTAAGGT-3' \\
U6 & 5'-CAAGCAACGTTGTTTAA-3' & 5'-TAGTTAAACAACGTTGCTTC-3' \\
GAPDH & 5'-ACAACTTTGGTATCGTGGAAGG-3 & 5'-GCCATCACGCCACAGTTTC-3' \\
\hline
\end{tabular}

Table 3. The sequence of primers for dual luciferase reporter gene assay

\begin{tabular}{ll}
\hline Name & Primer sequence \\
\hline NOX4 WT & F: 5'-AAACTCGAGTGCCATGAAGCAGGACTCTA-3' \\
NOX4 WT & R: 5'-AAAGCGGCCGCCCCCAGAGAAATAATCCCTCA-3' \\
NOX4 mut & F: 5'-TTAATTTGCACTTTGAGACCAATCGACATCATGTGTGTCAGTAGG-3' \\
NOX4 mut & R: 5'-CCTACTGACACACATGATGTCGATTGGTCTCAAAGTGCAAATTAA-3' \\
\hline
\end{tabular}


nant vectors and miR-mimics or miR-NC, respectively. Dual luciferase report gene assay system was used to measure the relative luciferase activity after 48 hours of culture. Each experiment was repeated 3 times and the results were represented as the mean \pm standard deviation.

3-(4,5-dimethylthiazol-2-yl)-2,5-diphenyltetrazolium bromide (MTT) assay. The 96-well plates were used for the culturing of cells in 6 groups $\left(3 \times 10^{3}\right.$ cells each group). Every transfection was done in triplicate. Every 24 hours $(0$ h, 24 h, $48 \mathrm{~h}, 72 \mathrm{~h}$ ), one of the plates was taken out of the incubator and each well was added with $20 \mu \mathrm{L}$ MTT $(5 \mathrm{mg} / \mathrm{mL})$, which was then put back into the incubator for 4 hours. Later, $150 \mu \mathrm{L}$ DMSO was added into the wells to dissolve its sediment afar the nutrient solution was removed. Then, the absorbance of each well at $560 \mathrm{~nm}$ was examined using spectrophotometer. Based on the $0 \mathrm{~h}$ absorbance, the cells viabilities after transfection for $24 \mathrm{~h}, 48 \mathrm{~h}$ and $72 \mathrm{~h}$ were calculated and recorded.

Colony formation assay. The transfected cells trypsinised and suspended in RPMI 1640 medium containing $10 \%$ FBS. Then, they were seeded in the 6-well plates with a density of 500 cells/well and had been cultured in the normal conditions after the sufficient vibration until they were visible to the naked eye. After $4 \%$ paraformaldehyde and crystal violet were used to fix for 15 minutes and stained for 20 minutes successively, the cell colonies were numerated with a microscope.

The flow cytometry (FCM). Forty-eight hours after transfection, the washed cells were fixed in $75 \%$ ethanol at $-20^{\circ} \mathrm{C}$ in a refrigerator. They were centrifuged and stored in a $4^{\circ} \mathrm{C}$ refrigerator overnight. Then, the ethanol was discarded and PBS was used to wash the cells. Later, the sediment cells were blended with DNA PrepTM (the fluorescent reagent) and incubated in dark for $20 \mathrm{~min}$. Cell cycle in each group was analyzed using FCM and ModFit LT. Additionally, based on the manufacturer's instructions, the other transfected cells were put in ice bath away from light. The Annexin V-FITC/ PI reagent was then added into the suspension. FCM was used to assess the apoptosis of cells.

Wound healing assay. TSCC 1 cells migration was assessed by the wound healing assay. The transfected cells were seeded into the 6-well plates until the cell confluence reached $100 \%$. The tip of a sterile micropipette was used to straightly scratch the cell surface. Following $24 \mathrm{~h}$ of incubation, the healing closure of the scratches was observed and photographed under a phase contrast microscopy and later the migration rates were calculated.

Transwell assay. Cell invasion was measured using the Transwell assay. The upper chambers were seeded with the suspended cells, meanwhile RPMI-1640 containing 10\%FBS were added into the lower chambers. Then, the chambers were placed into the incubator at $37^{\circ} \mathrm{C}$ with $5 \% \mathrm{CO}_{2} .24$ hours later, the liquor and the non-metastasis cells on the upper surface of the upper chambers were discarded. 10\% PBS was used to wash the cells, which were then fixed in $4 \%$ formalin for $15 \mathrm{~min}$ and dyed using crystal violet for $15 \mathrm{~min}$. The average invasive cells were counted in 10 random fields under a microscope.
Western blot analysis. Forty-eight hours after transfection, western blot was performed to examine the protein expression of the cells in different groups. Cell lysate extracted with trypsin was centrifuged under $12000 \mathrm{rpm}$ at $4^{\circ} \mathrm{C}$ for $30 \mathrm{~min}$ and later the concentration of protein was determined using the BCA method. Subsequently, total protein extracts were separated using $10 \%$ SDS-PAGE and transferred onto a PVDF membrane, which was then blocked with NOX4 primary antibodies in TBS-T containing $5 \%$ defatted milk at $4{ }^{\circ} \mathrm{C}$ overnight and the next day the second antibodies were added to the washed membrane. Eventually, the developed membrane was photographed using a microscope.

Statistical analysis. The normally distributed measurement data were analyzed with Student's t-test. The non-normally distributed measurement data were analyzed using one-way ANOVA and Rank sum test. Chi-square test was used to analyze the enumeration data. The measurement data were presented as mean \pm standard deviation. SPSS 19.0 was used for statistical analysis and the diagram was made using GraphPad Prism 6.0. $P$ values $<0.05$ were designed statistically significant.

\section{Results}

Down-expression of miR-99a-5p in human oral tumor. QRT-PCR was used to assess the expression level of miR99a-5p in TSCC1 cells and adjacent tissues. Compared with adjacent tissues, TSCC 1 cells expressed significantly less miR-99a-5p (Figure 1A), which implied that miR-99a-5p was down-expressed in human oral tumor.

Over-expression of NOX4 in human oral tumor. As Figure $1 \mathrm{~B}$ suggested, NOX4 mRNA was expressed noticeably more in TSCC1 cells than the adjacent tissues. Similarly, to compare the NOX4 protein expression in tumorous and adjacent tissues, we used western blot and Immunohistochemical analysis to measure the NOX4 protein in two groups and found that NOX4 protein was dramatically up-regulated in tumor tissues than adjacent tissues (Figure 1C-D). Collectively, NOX4 was over-expression in human oral tumor.

MiR-99a-5p targeted NOX4 in TSCC1 cells. To determine that NOX4 was a potential target gene of miR-99a-5p, the complimentary sequences of miR-99a-5p and NOX4 3'UTR were predicted by Targetscan and illustrated in Figure 2A. The recombinant plasmids, pGLO-wt and pGLO-mut, and pGLO-Null were co-transfected with miR mimics or miR mimics NC. MiR-99a-5p mimics+pGLO-NOX4-3'UTR (wt) group showed dramatically decreased luciferase activity than the corresponding control groups (Figure 2B). Consistent with above results, the over-expression of miR-99a-5p obviously reduced the NOX4 protein expression in comparison with the NC group, which further demonstrated that miR-99a-5p negatively modulated NOX4 gene expression by binding to the 3'UTR of NOX4 (Figure 2C).

Cell viability was assessed by MTT assay. MTT assay revealed that miR mimics and NOX4 siRNA groups showed 

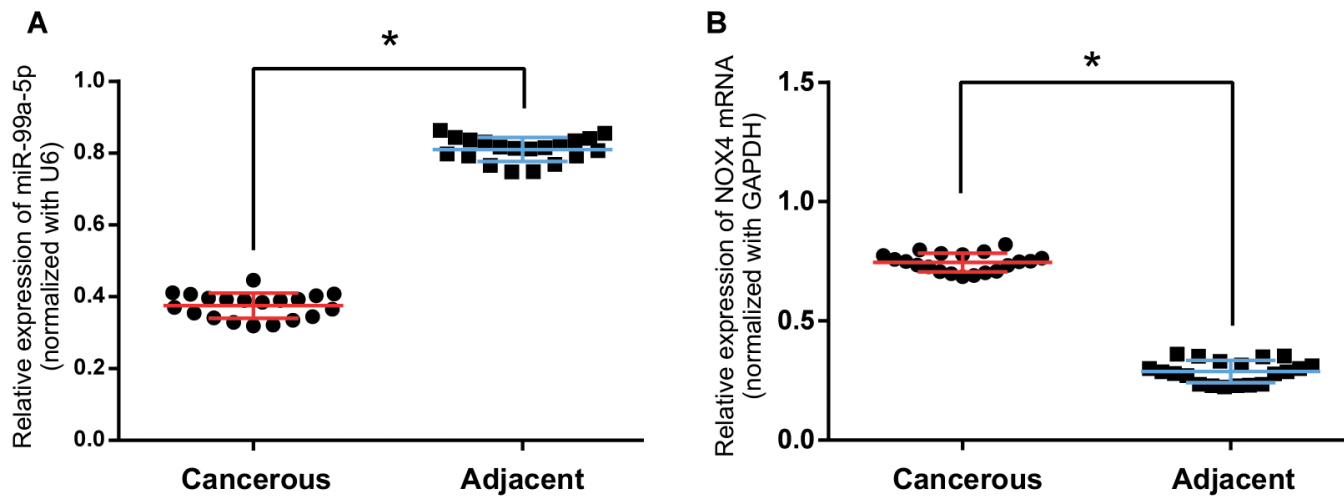

C

D

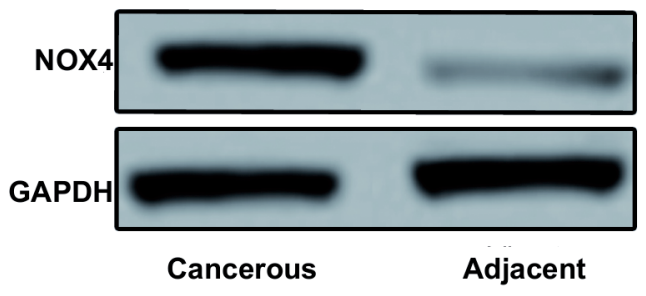

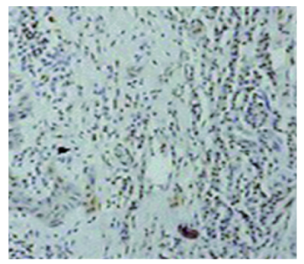

Cancerous

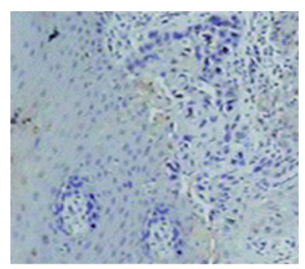

Adjacent

Figure 1. The expression of miR-99a-5p and NOX4 in human oral tumor and the adjacent tissues. (A) The relative expression level of miR-99a-5p in both TSCC1 cell line and adjacent tissues. (B) The relative expression level of NOX4 in both TSCC1 cell line and adjacent tissues. (C) NOX4 expression as revealed by western blot analysis in the oral tumor and adjacent tissues. ${ }^{\star} P$ values $<0.05$ were considered statistically significant.

a significant decrease in cell viability in contrast with the control and NC groups $(P<0.05)$, illustrating that the overexpression of miR-99a-5p or the down-expression NOX4 repressed the viability of tumor cells. Nevertheless, the viability of TSCC1 cells co-transfected with miR-99a-5p mimics and NOX4 cDNAs was dramatically higher than that of miR mimics and NOX4 siRNA groups (Figure 3A). These results showed that the inhibition of cell viability was achieved by the known-down of NOX 4 by the over-expression of miR-99a-5p.

Cell proliferation capacities were examined using colony formation assay. The effect of miR-99a-5p on cell proliferation was assessed using colony formation assay (Figure $3 \mathrm{~B}$ ). The proliferation of cells in miR mimics group showed a noticeable decrease comparing with the NC group. However, the colony formation rate of the cells co-transfected with both miR-99a-5p and NOX4 cDNAs was notably more than that of TSCC1 cells transfected with only miR mimics. In addition, the down-regulation of NOX4 in TSCC1 cells significantly declined the proliferation in contrast with the relevant NC group. Together, miR-99a-5p suppressed the proliferation of TSCC1 cells by down-regulating NOX4 expression.

Cell cycle and apoptosis levels were assessed using FCM. FCM results showed that compared with the control group, the proportion of $S$ phase cells declined significantly, while the
A

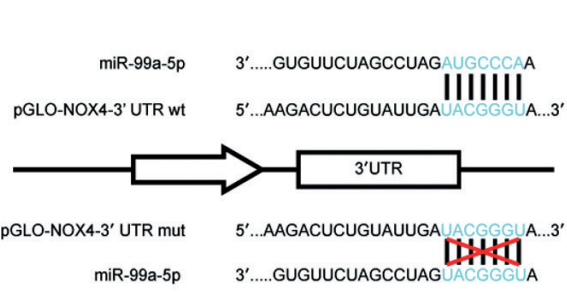

B

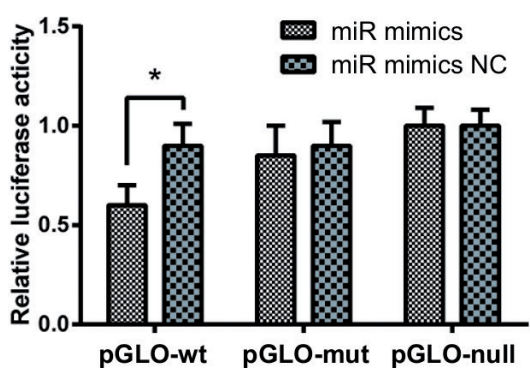

C

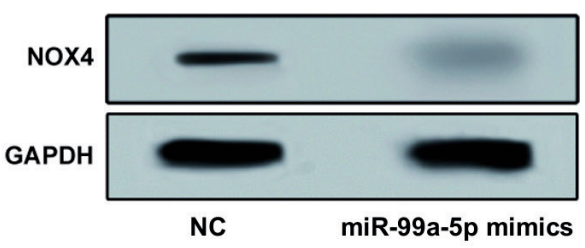

Figure 2. MiR-99a-5p directly targeted NOX4 in TSCC1 cells. (A) The sequences of pGLO-NOX4-3'UTR (wt), miR-99a-5p and pGLO-NOX4-3'UTR (mut). (B) Relative luciferase activity in wild-type and mutated recombinant vectors. (C) Western blot was performed to detect NOX4 expression of the cells transfected with miR-99a-5p mimics. ${ }^{\star} P$ values $<0.05$ were considered statistically significant. 


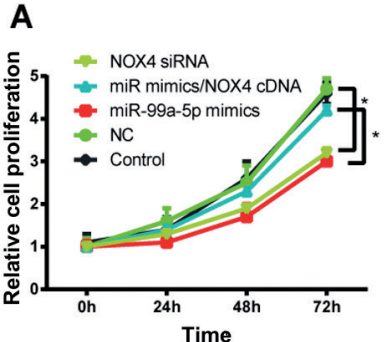

B

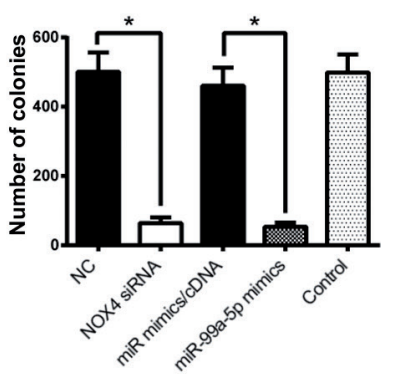

C

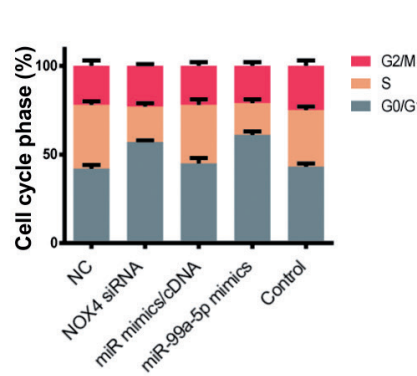

D

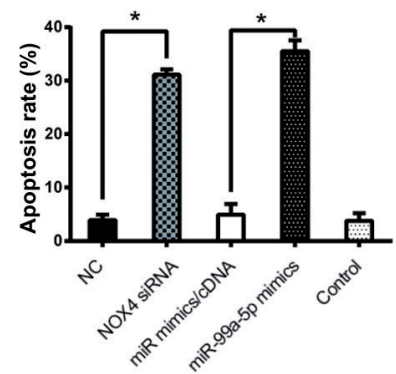


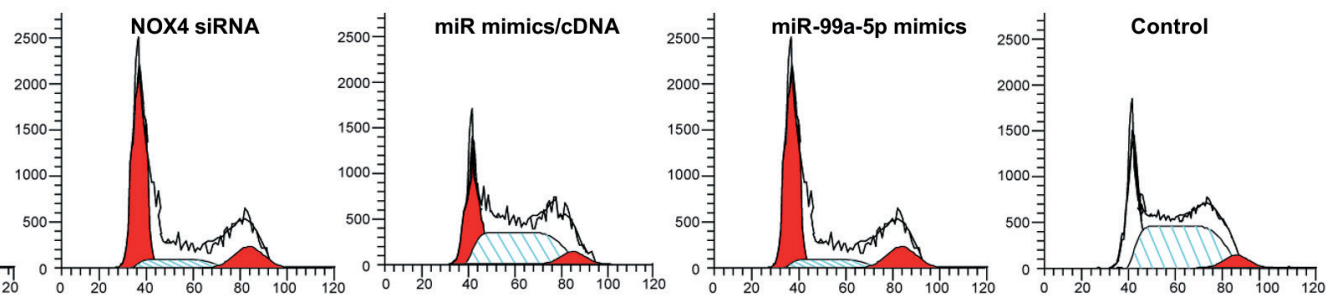

$\mathbf{F}$
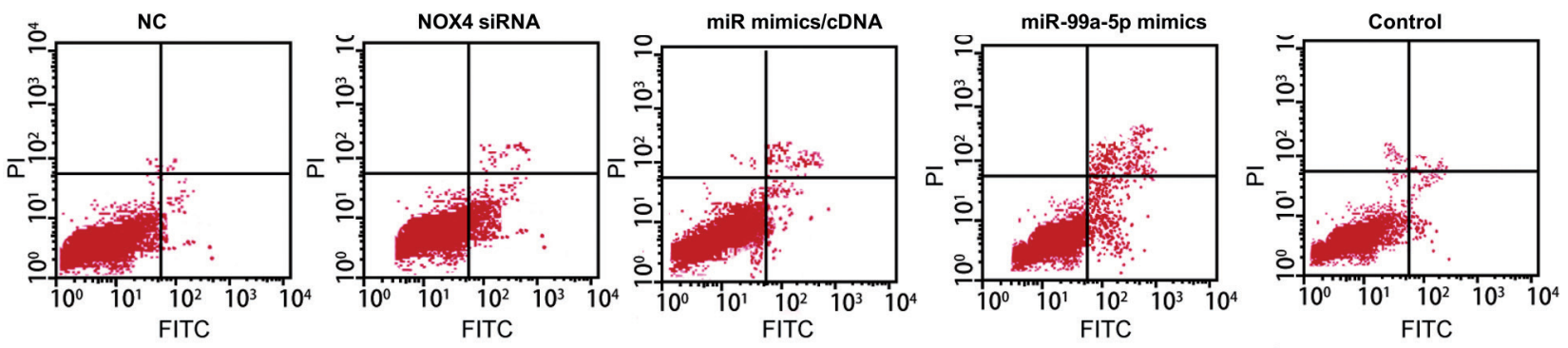

G
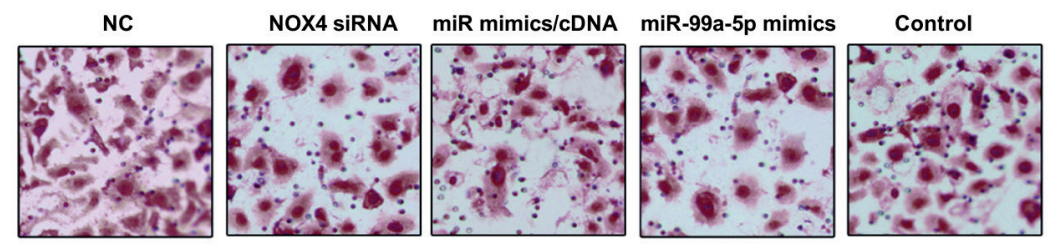

H

NC
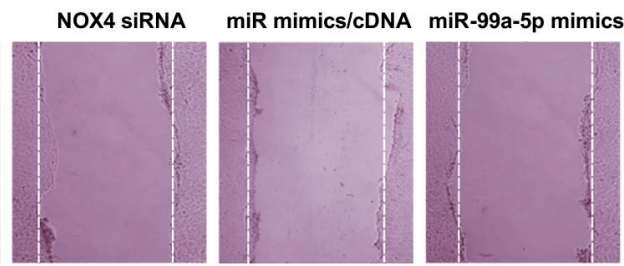

Control
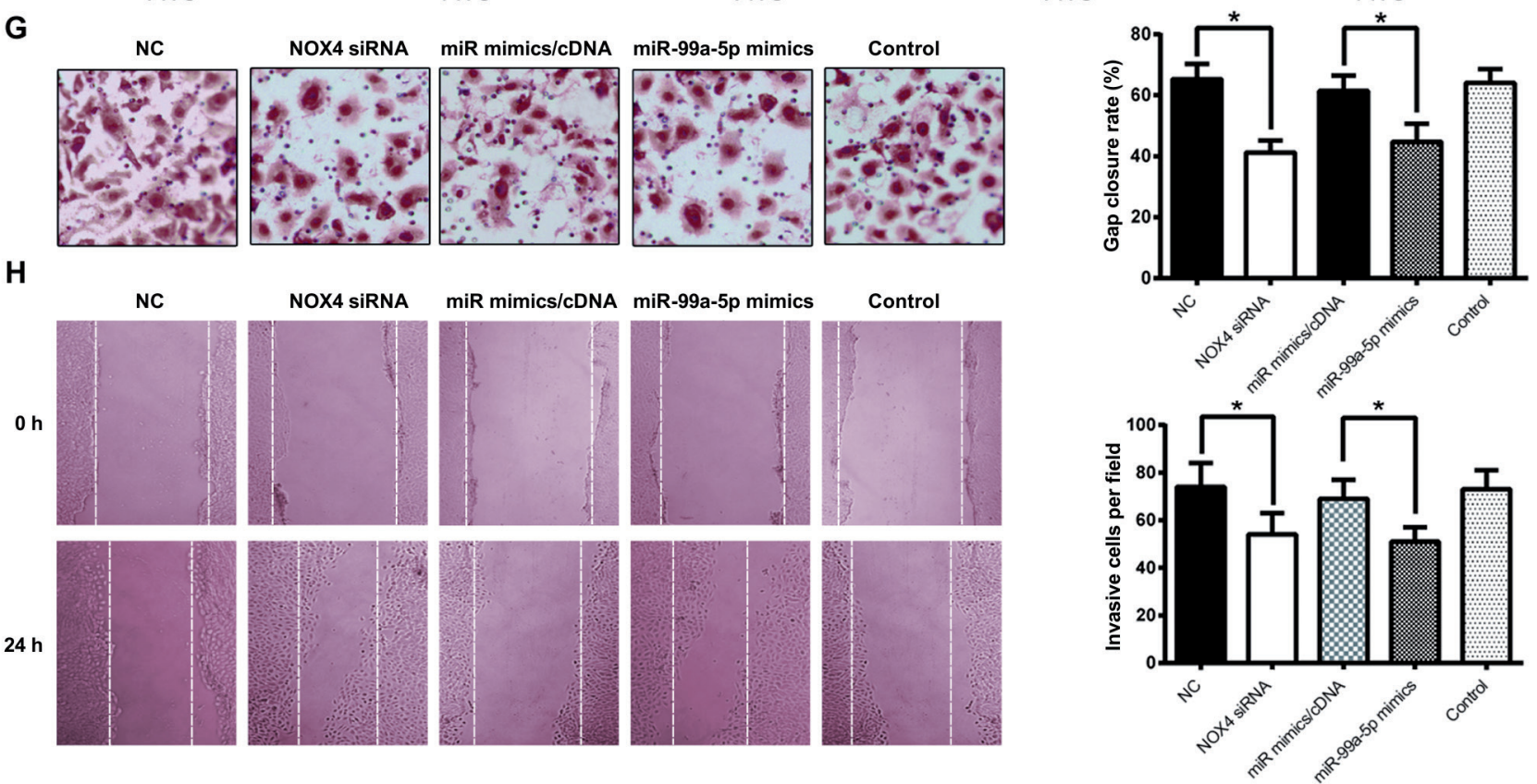

Figure 3. MiR-99a-5p hindered the viability, mitosis, migration and invasion of TSCC1 cells by negatively regulating NOX4. (A) MTT assay was performed to determine the cell viability in different transfection groups. (B) Colony formation assay was conducted to detect the proliferation of cells transfected with miR-mimics, NOX4 siRNA, miR mimics/cRNA and the empty vector. The histogram showed the number of colonies in every group. (C, E) FCM was conducted to investigate the cell cycle in different groups. (D, F) The apoptosis rate of the transfected cells in each group examined using FCM. (G) Transwell assay was performed to determine the invasion of the transfected cells in the NC, NOX4 siRNA, miR mimics/cRNA, miR mimics and control group. ${ }^{\star} P$ values $<0.05$ were considered statistically significant. $(\mathrm{H})$ Differences in the cell migration of transfected cell lines confirmed by wound healing assay. 
proportion of G0/G1 phase cells increased obviously in miR mimics group (Figure 3C, E), which implied that miR-99a-5p mimics arrested TSCC1 cells in G0/G1 phase. Likewise, cells in the NOX4 siRNA group also showed a similar proportion of $S$ phase cells with miR mimics group, whereas the $S$ phase TSCC1 cells in miR mimics/NOX4 cDNA group significantly outnumbered the miR mimics and NOX4 siRNA groups. What's more, the apoptotic cells were obviously more in miR$99 a-5 p$ mimics group than in the control group. However, the apoptosis in miR mimics/cDNA group declined strongly comparing with the miR mimics group, while NOX4 siRNA group showed a dramatical increase of apoptosis (Figure 3D, F).

Cell invasion was investigated by transwell assay. We used transwell assay to assess cell invasiveness. In contrast with the number of invasive cells in the NC group, it was dramatically less in miR mimics group. Similarly, the number of invaded cells in the NOX4 siRNA group was also substantially less than the NC group. The simultaneous over-expression of NOX4 and miR-99a-5p promoted the cell invasion comparing with cells transfected with miR-99a-5p mimics (Figure 3G).

Cell migration was examined using wound healing assay. The results of wound healing assay were shown in Figure $3 \mathrm{H}$. After the transfection of miR mimics, the healing level was noticeably decreased compared with the NC group. In addition, the down-expression of NOX4 in TSCC1 cells also obviously suppressed the wound healing level. Therefore, the data above suggested that cell migratory abilities were hindered by the over-expression of miR-99a-5p or the down-expression of NOX4. Moreover, when the cells were co-transfected with exogenous NOX4 and miR-99a-5p, the cell migration was more severe than the miR-99a-5p group.

\section{Discussion}

In the present study, we primarily investigated the effects of miR-99a-5p on human oral carcinoma cell activities by suppressing NOX4. We found that miR-99a-5p was underexpressed whereas NOX4 was over-expressed in human tumorous tissues and cell lines. MiR-99a-5p significantly retarded the viability, proliferation, migration, mitosis and invasiveness but promoted the apoptosis of oral cancer cells by directly binding to NOX4 3'UTR.

Bioinformatics approaches have been applied to determine the deregulated miRNAs and NOX4 were associated with molecular pathways that are involved in tumorigenesis [1114]. MiR-99a-5p was found down-regulated in human oral cancerous tissues, which suggests its possible tumor suppressor role in human oral cancer. To the contrary, NOX4 was found up-regulated in human oral cancerous tissues, suggesting its possible oncogenic role in human oral cancer. Our findings are consistent with those of Aoyama's that SOD1mu incudedNOX1 activation increased NOX4 level during hepatic fibrosis or carcinogenesis and the target-down of NOX1/4 could be a possible novel therapy for hepatic fibrosis and tumorigenesis [15-17]. Besides, Thian-Sze et al. discovered that a plethora of miRNAs were significantly aberrantly expressed in human tongue squamous cell carcinoma tissues, among which miR-99a was 3-fold less expressed compared with the normal tissues [18]. Not coincidently, miR-99a was found significantly down-regulated in various cancers including OSCC and head and neck squamous cell carcinoma (HNSCC) [19-21]. The natural duties of miRNA are to degrade or stabilize target mRNAs by binding to their 3'UTRs, which then affected the cell activities such as proliferation and apoptosis. For instance, miR-99a was found hindered the progression to melanoma by down-regulating mTOR and IGF1R signaling. Similarly, miR-99a/b were found down-regulated in human cervical cancerous tissues and their overexpression could suppress the carcinogenesis by repressing mTOR. The activation of mTOR signaling (mTORC1 and mTORC2/Akt) has been reported to induce melanoma formation [22]. MiR-99a/b could negatively regulate mTOR, which then retard human cervical carcinogenesis [23]. In addition, miR-99a was also found to inhibit mTOR/NF-kB signaling in endothelial cell inflammation and endometrioid endometrial carcinoma [24]. Of our interest, NOX4 was found directly targeted by miR99a in lung adenocarcinoma [25]. NOX4 encodes a catalytic subunit of NADPH oxidases, which catalyze the reduction of molecular oxygen to various reactive oxygen species (ROS). ROS has been reported to participate in cell differentiation and tumor growth. It has been believed that NOX4 is related with fibrosis and idiopathic $[16,26]$. Also, NOX family has been implicated in melanoma aggressiveness. For instance, Ito et al. found that NOX1/4 were both highly expressed in OSCC cell lines and the knock-down of NOX1 or the inhibition of AKT led to the reduced cell viability and mitosis in OSCC cells [27]. NOX4 can be inhibited by fulvene-5 in hemangioma, which then retarded the growth of hemangioma in vivo [28]. Besides, the knockdown of NOX2/4 led to the reduction of hematopoietic cell proliferation and migration transformed by TKOs or TGF $\beta 1[17,29]$. Furthermore, NOX4 is closely associated with PI3K/AKT signaling. For example, AKT can induce NOX4 expression and serves as a positive molecular switch of angiogenesis and melanoma aggressiveness [15]. Thus, the aberrant expression of miR-99a and NOX4 are commonly seen in various cancers and can be potential carcinogenesis biomarkers. MiR-99a-5p was confirmed to fulfill its 'duty' in oral cancer cells by directly binding to NOX4 3'UTR in the present study. Exogenous miR-99a-5p substantially reduced the aggressiveness of oral squamous carcinoma cells by suppressing NOX4.

However, miR-99a could also be a potential onco-miRNA in pediatric myeloid leukemia by suppressing CTDSPL and TRIB2, which are tumor suppressors [30], and they were also found highly-expressed in human acute megakaryoblastic leukemia by altering the balance between TGF $\beta$ and Wnt signaling [31]. We believe that miRNAs can be either tumor suppressive or oncogenic, which is dependent on the organ or tissue context. Besides, we found that NOX4 was involved in a series of signaling pathways that could explain 
the mechanism through which it influences cancer development. The molecular mechanism thus needs extensive study. Intriguingly, recent studies have reported that fusion genes could escape the regulation of miRNA by deleting its 3'UTR. Fusion genes are caused by tandem duplication on chromosomes, which have been found in various cancer and promote tumorigenesis. For instance, FGFR3 has been found lack of 3'UTR, which released it from miR-99a-dependent suppression and induced glioblastoma carcinogenesis [32, 33]. Thus, a thorough investigation in tumorigenesis network could always assist with choosing the precise target therapy in practice.

In summary, in the present study, we explored the deregulation of miR-99a-5p and NOX4 in OSCC cells. The interplay of miR-99a-5p and NOX4 affected OSCC cell activities and could be a novel target for OSCC treatment.

\section{References}

[1] TANG H, WU Z, ZHANG J, SU B. Salivary lncRNA as a potential marker for oral squamous cell carcinoma diagnosis. Mol Med Rep 2013; 7: 761-766.

[2] CHOI S, MYERS JN. Molecular pathogenesis of oral squamous cell carcinoma: implications for therapy. J Dent Res 2008; 87: 14-32. https://doi.org/10.1177/154405910808700104

[3] ROGERS SN, BROWN JS, WOOLGAR JA, LOWE D, MAGENNIS $P$ et al. Survival following primary surgery for oral cancer. Oral Oncol 2009; 45: 201-211. https://doi. org/10.1016/j.oraloncology.2008.05.008

[4] NILSEN TW. Mechanisms of microRNA-mediated gene regulation in animal cells. Trends Genet 2007; 23: 243-249. https://doi.org/10.1016/j.tig.2007.02.011

[5] TIWARI A, SHIVANANDA S, GOPINATH KS, KUMAR A. MicroRNA-125a reduces proliferation and invasion of oral squamous cell carcinoma cells by targeting estrogen-related receptor alpha: implications for cancer therapeutics. J Biol Chem 2014; 289: 32276-32290. https://doi.org/10.1074/jbc. M114.584136

[6] SHI W, YANG J, LI S, SHAN X, LIU X et al. Potential involvement of miR-375 in the premalignant progression of oral squamous cell carcinoma mediated via transcription factor KLF5. Oncotarget 2015; 6: 40172-40185. https://doi. org/10.18632/oncotarget.5502

[7] KUO YZ, TAI YH, LO HI, CHEN YL, CHENG HC et al. MiR-99a exerts anti-metastasis through inhibiting myotubularin-related protein 3 expression in oral cancer. Oral Dis 2014; 20: e65-75. https://doi.org/10.1111/odi.12133

[8] FU Y, ZHANG Y, WANG Z, WANG L, WEI X et al. Regulation of NADPH oxidase activity is associated with miRNA25-mediated NOX4 expression in experimental diabetic nephropathy. Am J Nephrol 2010; 32: 581-589. https://doi. org/10.1159/000322105

[9] IM YB, JEE MK, CHOI JI, CHO HT, KWON OH et al. Molecular targeting of NOX4 for neuropathic pain after traumatic injury of the spinal cord. Cell Death Dis 2012; 3: e426. https:// doi.org/10.1038/cddis.2012.168
[10] CROSAS-MOLIST E, BERTRAN E, SANCHO P, LOPEZLUQUE J, FERNANDO J et al. The NADPH oxidase NOX4 inhibits hepatocyte proliferation and liver cancer progression. Free Radic Biol Med 2014; 69: 338-347. https://doi. org/10.1016/j.freeradbiomed.2014.01.040

[11] MANIKANDAN M, DEVA MAGENDHRA RAO AK, ARUNKUMAR G, MANICKAVASAGAM M, RAJKUMAR KS et al. Oral squamous cell carcinoma: microRNA expression profiling and integrative analyses for elucidation of tumourigenesis mechanism. Mol Cancer 2016; 15: 28. https://doi. org/10.1186/s12943-016-0512-8

[12] VICTORIA MARTINEZ B, DHAHBI JM, NUNEZ LOPEZ YO, LAMPERSKA K, GOLUSINSKI P et al. Circulating small non-coding RNA signature in head and neck squamous cell carcinoma. Oncotarget 2015; 6: 19246-19263. https://doi. org/10.18632/oncotarget.4266

[13] FUKUMOTO I, HANAZAWA T, KINOSHITA T, KIKKAWA $\mathrm{N}$, KOSHIZUKA K et al. MicroRNA expression signature of oral squamous cell carcinoma: functional role of microRNA$26 \mathrm{a} / \mathrm{b}$ in the modulation of novel cancer pathways. Br J Cancer 2015; 112: 891-900. https://doi.org/10.1038/bjc.2015.19

[14] BOLDRUP L, COATES PJ, LAURELL G, WILMS T, FAHRAEUS R et al. Downregulation of miRNA-424: a sign of field cancerisation in clinically normal tongue adjacent to squamous cell carcinoma. Br J Cancer 2015; 112: 1760-1765. https://doi.org/10.1038/bjc.2015.150

[15] GOVINDARAJAN B, SLIGH JE, VINCENT BJ, LI M, CANTER JA et al. Overexpression of Akt converts radial growth melanoma to vertical growth melanoma. J Clin Invest 2007; 117: 719-729. https://doi.org/10.1172/JCI30102

[16] AOYAMA T, PAIK YH, WATANABE S, LALEU B, GAGGINI $F$ et al. Nicotinamide adenine dinucleotide phosphate oxidase in experimental liver fibrosis: GKT137831 as a novel potential therapeutic agent. Hepatology 2012; 56: 2316-2327. https:// doi.org/10.1002/hep. 25938

[17] SENTURKS, MUMCUOGLU M, GURSOY-YUZUGULLUO, CINGOZ B, AKCALI KC et al. Transforming growth factorbeta induces senescence in hepatocellular carcinoma cells and inhibits tumor growth. Hepatology 2010; 52: 966-974. https:// doi.org/10.1002/hep.23769

[18] WONG TS, LIU XB, WONG BY, NG RW, YUEN AP et al. Mature miR-184 as Potential Oncogenic microRNA of Squamous Cell Carcinoma of Tongue. Clin Cancer Res 2008; 14: 2588-2592. https://doi.org/10.1158/1078-0432.CCR-07-0666

[19] YEN YC, SHIAH SG, CHU HC, HSU YM, HSIAO JR et al. Reciprocal regulation of microRNA-99a and insulin-like growth factor I receptor signaling in oral squamous cell carcinoma cells. Mol Cancer 2014; 13: 6. https://doi.org/10.1186/14764598-13-6

[20] CHEN D, CABAY RJ, JIN Y, WANG A, LU Y et al. MicroRNA Deregulations in Head and Neck Squamous Cell Carcinomas. J Oral Maxillofac Res 2013; 4: e2. https://doi.org/10.5037/ jomr.2013.4102

[21] CHEN Z, JIN Y, YU D, WANG A, MAHJABEEN I et al. Downregulation of the microRNA-99 family members in head and neck squamous cell carcinoma. Oral Oncol 2012; 48: 686-691. https://doi.org/10.1016/j.oraloncology.2012.02.020 
[22] DAMSKY W, MICEVIC G, MEETH K, MUTHUSAMY V, CURLEY DP et al. mTORC1 activation blocks BrafV600E-induced growth arrest but is insufficient for melanoma formation. Cancer Cell 2015; 27: 41-56. https://doi.org/10.1016/j. ccell.2014.11.014

[23] WANG L, CHANG L, LI Z, GAO Q, CAI D et al. miR-99a and -99b inhibit cervical cancer cell proliferation and invasion by targeting mTOR signaling pathway. Med Oncol 2014; 31: 934. https://doi.org/10.1007/s12032-014-0934-3

[24] TORRES A, TORRES K, PESCI A, CECCARONI M, PASZKOWSKI T et al. Deregulation of miR-100, miR-99a and miR-199b in tissues and plasma coexists with increased expression of mTOR kinase in endometrioid endometrial carcinoma. BMC Cancer 2012; 12: 369. https://doi. org/10.1186/1471-2407-12-369

[25] SUN M, HONG S, LI W, WANG P, YOU J et al. MiR-99a regulates ROS-mediated invasion and migration of lung adenocarcinoma cells by targeting NOX4. Oncol Rep 2016; 35 : 2755-2766. https://doi.org/10.3892/or.2016.4672

[26] BETTAIEB A, JIANG JX, SASAKI Y, CHAO TI, KISS Z et al. Hepatocyte Nicotinamide Adenine Dinucleotide Phosphate Reduced Oxidase 4 Regulates Stress Signaling, Fibrosis, and Insulin Sensitivity During Development of Steatohepatitis in Mice. Gastroenterology 2015; 149: 468-480.e410. https://doi. org/10.1053/j.gastro.2015.04.009

[27] ITO K, OTA A, ONO T, NAKAOKA T, WAHIDUZZAMAN $\mathrm{M}$ et al. Inhibition of Noxl induces apoptosis by attenuating the AKT signaling pathway in oral squamous cell carci- noma cell lines. Oncol Rep 2016; 36: 2991-2998. https://doi. org/10.3892/or.2016.5068

[28] BHANDARKAR SS, JACONI M, FRIED LE, BONNER MY, LEFKOVE B et al. Fulvene-5 potently inhibits NADPH oxidase 4 and blocks the growth of endothelial tumors in mice. J Clin Invest 2009; 119: 2359-2365. https://doi.org/10.1172/ LCI33877

[29] REDDY MM, FERNANDES MS, SALGIA R, LEVINE RL, GRIFFIN JD et al. NADPH oxidases regulate cell growth and migration in myeloid cells transformed by oncogenic tyrosine kinases. Leukemia 2011; 25: 281-289. https://doi.org/10.1038/ $\underline{\text { leu. } 2010.263}$

[30] ZHANG L, LI X, KE Z, HUANG L, LIANG Y et al. MiR-99a may serve as a potential oncogene in pediatric myeloid leukemia. Cancer Cell Int 2013; 13: 110. https://doi. org/10.1186/1475-2867-13-110

[31] EMMRICH S, RASCHE M, SCHONING J, REIMER C, KEIHANI $S$ et al. miR-99a/100 125b tricistrons regulate hematopoietic stem and progenitor cell homeostasis by shifting the balance between TGFbeta and Wnt signaling. Genes Dev 2014; 28: 858-874. https://doi.org/10.1101/gad.233791.113

[32] BABIC I, MISCHEL PS. Multiple functions of a glioblastoma fusion oncogene. J Clin Invest 2013; 123: 548-551. https://doi. org/10.1172/JCI67658

[33] PARKER BC, ANNALA MJ, COGDELL DE, GRANBERG KJ, SUN Y et al. The tumorigenic FGFR3-TACC3 gene fusion escapes miR-99a regulation in glioblastoma. J Clin Invest 2013; 123: 855-865. https://doi.org/10.1172/JCI67144 